\title{
IncRNA ZFAS1 promotes the ox-LDL induced proliferation, invasion and migration of vascular smooth muscle cells
}

\author{
HAO WANG ${ }^{1}$, HUAJIE HU ${ }^{1}$, JUNJIE MA ${ }^{1}$, YAFENG JIANG ${ }^{2}$ and RUIFEI CHENG ${ }^{3}$ \\ ${ }^{1}$ Department of Clinical Laboratory, Linhai Traditional Chinese Medicine Hospital Medical Community, Linhai, \\ Zhejiang 317000; ${ }^{2}$ Department of Medical Genetics and Prenatal Diagnosis, Lianyungang Maternal and \\ Child Health Hospital, Lianyungang, Jiangsu 222000; ${ }^{3}$ Department of Clinical Laboratory, \\ Lianyungang Traditional Chinese Medicine Hospital Affiliated to Nanjing University \\ of Chinese Medicine, Lianyungang, Jiangsu 222004, P.R. China
}

Received October 24, 2020; Accepted March 18, 2021

DOI: 10.3892/etm.2021.10267

\begin{abstract}
Atherosclerosis is a chronic progressive inflammatory vascular disease. The dysfunction of vascular smooth muscle cells (VSMCs) induced by oxidized low-density lipoprotein (ox-LDL) contributes to the formation of atherosclerotic lesions. Additionally, upregulation of the long non-coding RNA zinc finger antisense 1 (ZFAS1) was observed in the plaques of patients with atherosclerosis. The aim of the present study was to explore the functional role of ZFAS1 in atherosclerosis progression. Reverse transcription-quantitative PCR was performed to analyze ZFAS1 mRNA expression, and western blotting was performed to determine the protein expression levels of Ki67, proliferating cell nuclear antigen (PCNA), matrix metallopeptidase (MMP)2 and MMP9. The Cell Counting Kit-8 assay was used to test cell viability. Finally, wound healing and Transwell chamber assays were performed to evaluate cell migration and invasion, respectively. The current findings demonstrated that ZFAS1 expression was upregulated by ox-LDL stimulation in VSMCs. Moreover, ZFAS1 overexpression promoted the ox-LDL-induced proliferation, migration and invasion of VSMCs, and upregulated the expression levels of proteins associated with cellular proliferation (Ki67 and PCNA), migration and invasion (MMP2 and 9). By contrast, ZFAS1-knockdown inhibited the proliferation, migration and invasion of VSMCs, and suppressed cell proliferation-, migration- and invasion-associated protein expression. In conclusion, ZFAS1 promoted the ox-LDL-induced proliferation, invasion and
\end{abstract}

Correspondence to: Dr Ruifei Cheng, Department of Clinical Laboratory, Lianyungang Traditional Chinese Medicine Hospital Affiliated to Nanjing University of Chinese Medicine, 160 Chaoyang Middle Road, Haizhou, Lianyungang, Jiangsu 222004, P.R. China E-mail: crf1704h@163.com

Key words: long non-coding RNA zinc finger antisense 1, oxidized low-density lipoprotein, proliferation, invasion, migration, vascular smooth muscle cells migration of VSMCs. Thus, ZFAS1 may represent a novel biomarker for dysfunction of VSMCs in the pathological condition of atherosclerosis.

\section{Introduction}

Atherosclerosis is a chronic progressive inflammatory vascular disease, which poses a major threat to human health and has been attracting increasing attention (1). Thrombotic events associated with acute rupture or erosion of an unstable plaque, rather than gradual narrowing of the lumen, have been shown to be responsible for the majority of the clinical consequences of atherosclerosis (2). It is well established that vascular smooth muscle cells (VSMCs) serve an important role in the pathogenesis of atherosclerosis $(2,3)$. VSMCs, as one of the key components of the plaques, are derived from the medial layer of the vessel wall, which acts as a regulator of the atherosclerotic plaque (4-6). Furthermore, excess proliferation or dysfunction of VSMCs contributes to atherogenesis as a response to vascular injury, inflammation and lipoprotein accumulation during disease progression $(1,7)$. Oxidized low-density lipoprotein (ox-LDL) contributes to the atherosclerotic lesion through several mechanisms, such as the dysfunction of endothelial cells, and the excess migration and proliferation of VSMCs, as well as contributing to plaque instability (8). Therefore, in the present study VSMCs were treated with ox-LDL and used as the cell model for atherosclerosis in order to investigate the specific mechanisms underlying the pathogenesis of atherosclerosis.

Previous studies have revealed that long non-coding RNAs (lncRNAs), a class of non-coding RNAs >200 nucleotides in length, serve an important role in the onset and development of multiple human diseases, including cancer, diabetes, inflammatory diseases and cardiovascular diseases (9-11). The expression levels of the lncRNA zinc finger antisense 1 (ZFAS1) were revealed to be upregulated in the plaques of patients with atherosclerosis compared with in controls, as well as in atherosclerosis rat models $(12,13)$, indicating that ZFAS1 is closely associated with the progression of atherosclerosis. A recent study reported that ZFAS1 upregulation was observed in the cytoplasm and sarcoplasmic reticulum of mouse 
cardiomyocytes challenged with hypoxic stimulation, and that it impaired cardiac function in a mouse model of acute myocardial infarction, and these effects were readily reversed by ZFAS1-knockdown (14). Furthermore, IncRNA ZFAS1 promotes the proliferation, invasion and migration of various cancer cells, including nasopharyngeal carcinoma (15), cervical carcinoma (16) and colorectal cancer (17). Additionally, ZFAS1 may promote the proliferation and migration of chondrocytes, and suppress apoptosis and matrix synthesis in osteoarthritis (18). Overall, the aforementioned findings indicate that ZFAS1 serves a key role in promoting cell proliferation and invasion. Thus, it was hypothesized that ZFAS1 may serve as a potential biomarker in atherosclerosis induced by ox-LDL, and may promote the proliferation and invasion of VSMCs under pathological conditions.

In the present study, VSMCs were treated with multiple doses of ox-LDL to induce a cell model of atherosclerosis, in order to investigate whether ZFAS1 expression was upregulated by ox-LDL treatment in a dose-dependent manner and to determine whether ZFAS1 may be of value as a novel biomarker for dysfunction of VSMCs in the pathological condition of atherosclerosis.

\section{Materials and methods}

Cell culture and transfection. The VSMC cell line was obtained from the China Infrastructure of Cell Line Resources, Institute of Basic Medical Sciences (Chinese Academy of Sciences). The cells were cultured in DMEM (HyClone; Cytiva) with $10 \%$ fetal bovine serum (FBS; Gibco; Thermo Fisher Scientific, Inc.), $1 \%$ penicillin $(100 \mathrm{U} / \mathrm{ml})$ and $1 \%$ streptomycin $(100 \mathrm{mg} / \mathrm{ml})$ (Beyotime Institute of Biotechnology) at $37^{\circ} \mathrm{C}$ in a humidified incubator with $5 \% \mathrm{CO}_{2}$. VSMCs were cultured in 6-well plates for $12 \mathrm{~h}$, and then transfected with indicated plasmids. ZFAS1-1 small interfering (si)RNA (5'-CTGGCT GAACCAGTTCCACAAGGTT-3'), ZFAS1-2 siRNA (5'-TAC TTCTCCTAGTTGCAGTCAGG-3') and the scramble negative control siRNA (si-NC; 5'-ACGTGACACGTTCGG AGAATT-3') were obtained from Shanghai GenePharma Co., Ltd., and $100 \mathrm{nM}$ of each siRNA was transfected into VSMCs for ZFAS1-knockdown using Lipofectamine ${ }^{\circledR} 2000$ (Invitrogen; Thermo Fisher Scientific, Inc.) following the manufacturer's instructions. In addition, ZFAS1 transcript cDNA (5'-UGCGUGCCAAGCGCGACAUGGCGCGGA AGCCGAGAAGCCCCGGAGGCCC-3') was inserted into the pCDNA3.1 vector [Jiman Biotechnology (Shanghai) Co., Ltd.] and constructed by Biotech Integrated Solutions, and then $2 \mathrm{mg} / 1$ ZGAS1 pcDNA3.1 or empty pcDNA3.1 was transfected into VSMCs to achieve ZFAS1 overexpression (oe-ZFAS1) using Lipofectamine ${ }^{\circledR} 2000$. After transfection for $8 \mathrm{~h}$ at $37^{\circ} \mathrm{C}$, the VSMCs were exposed to ox-LDL $(25,50$ and $100 \mathrm{mg} / \mathrm{l}$; Beijing Solarbio Science \& Technology Co., Ltd.) for 12,24 and $48 \mathrm{~h}$ at $37^{\circ} \mathrm{C}$.

$R N A$ isolation and reverse transcription-quantitative (RT- $q)$ $P C R$. Total RNA from VSMCs was isolated using TRIzol ${ }^{\circledR}$ reagent (Invitrogen; Thermo Fisher Scientific, Inc.) following the manufacturer's instructions. RNA ( $1 \mu \mathrm{g})$ was reverse transcribed into first-strand cDNA using the Reverse Transcriptase kit according to the manufacturer's protocol (TransGen Biotech Co., Ltd.).
qPCR was performed using SYBR Green Mixture (Takara Bio, Inc.) in the ABI Prism 7300 Sequence Detection System (Applied Biosystems; Thermo Fisher Scientific, Inc.). The thermocycling conditions were: Initial denaturation for $10 \mathrm{~min}$ at $94^{\circ} \mathrm{C}$, followed by 40 of cycles of denaturation for $30 \mathrm{sec}$ at $94^{\circ} \mathrm{C}$ , annealing for $30 \mathrm{sec}$ at $55^{\circ} \mathrm{C}$ and extension for $30 \mathrm{sec}$ at $72^{\circ} \mathrm{C}$ The $2^{-\Delta \Delta \mathrm{Ca}}$ method (19) was applied to determine the relative target gene expression. The sequences for the qPCR primers were as follows: ZFAS1 forward, 5'-AGCGTTTGCTTT GTTCCC-3' and reverse, 5'-CTCCCTCGATGCCCTTCT-3'; GAPDH forward, 5'-GGTCTCCTCTGACTTCAACA-3' and reverse, 5'-AGCCAAATTCGTTGTCATAC-3'. GAPDH was employed as an internal control.

Cell Counting Kit-8 (CCK-8) assay. The cell viability of VSMCs was determined using the CCK-8 assay (Dojindo Molecular Technologies, Inc.). Briefly, VSMCs transfected with or without oe-ZFAS1 or si-ZFAS1 were seeded at the density of $2 \times 10^{3}$ cells/well into 96 -well plates and treated with ox-LDL. After transfection for 12, 24 and 48 h, CCK-8 reagent $(10 \mu \mathrm{l})$ was added into each well and incubated with VSMCs for another $2 \mathrm{~h}$. The absorbance at $450 \mathrm{~nm}$ was measured using an ELISA plate reader (Bio-Rad Laboratories, Inc.).

Western blotting. After treatment, proteins were isolated from VSMCs transfected with or without oe-ZFAS1 or si-ZFAS1 using RIPA lysis buffer (Beyotime Institute of Biotechnology) and quantified using a BCA assay kit (Thermo Fisher Scientific, Inc.). Protein samples ( $25 \mu \mathrm{g} / \mathrm{lane})$ were loaded and separated via 10\% SDS-PAGE and transferred onto PVDF membranes (EMD Millipore). After blocking with 5\% skimmed milk for $1 \mathrm{~h}$ at room temperature, the membrane was incubated with primary antibodies against Ki67 (cat. no. ab21700; 1:1,000; Abcam), proliferating cell nuclear antigen [PCNA; cat. no. 13110; 1:1,000; Cell Signaling Technology, Inc. (CST)], matrix metallopeptidase (MMP)2 (cat. no. 4022; 1:1,000; CST), MMP9 (cat. no. 3852; 1:1,000; CST) and GAPDH (cat. no. 8884; 1:2,000; CST) overnight at $4^{\circ} \mathrm{C}$. After washing with PBS, the membrane was incubated with horseradish peroxidase-conjugated secondary antibody (cat. no. ab97080; 1:10,000; Abcam) for $2 \mathrm{~h}$ at room temperature. ECL Reagent was used to develop color of protein bands (SuperSignal West Atto; Thermo Fisher Scientific, Inc). The gray values of the protein bands were determined using ImageJ software (version 1.48; National Institutes of Health). Wound healing assay. Wound healing assay was performed to assess the migration of VSMCs. Cells were plated in 6-well plates to generate a confluent monolayer. A scratch was created using a 200- $\mu l$ sterile pipette tip, followed by washing with PBS three times. Subsequently, VSMCs were cultured with fresh serum-free DMEM for $24 \mathrm{~h}$ at $37^{\circ} \mathrm{C}$. Finally, the width of the scratch wound was observed, and images were captured at x100 magnification using a fluorescence microscope (Olympus IX53; Olympus Corporation). The recovered wound area (\%) at the indicated time point $(24 \mathrm{~h})$ was calculated according to the following formula: [(wound width at $0 \mathrm{~h}$ ) - (wound width at $24 \mathrm{~h}$ )] / wound width at $0 \mathrm{~h}$.

Transwell chamber assay. The Transwell chamber assay was used to determine the invasive ability of VSMCs. Briefly, 

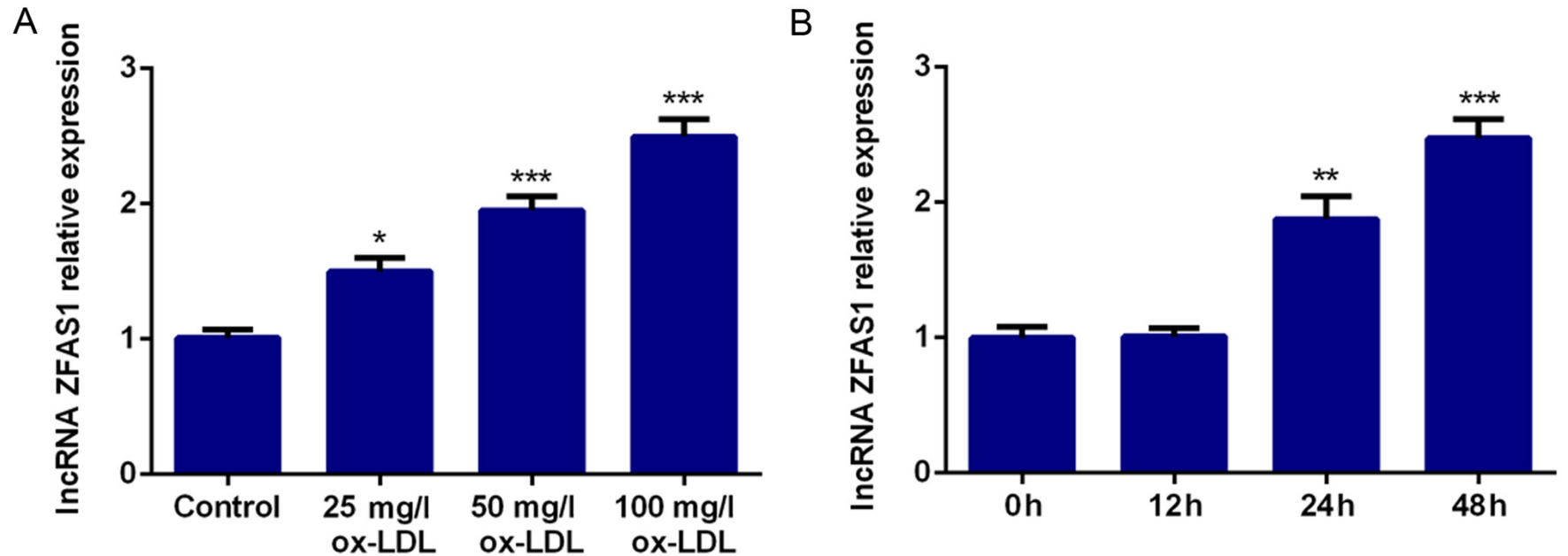

Figure 1. ZFAS1 expression is upregulated by ox-LDL treatment in VSMCs. Relative ZFAS1 mRNA expression was quantified via reverse transcription-quantitative PCR in VSMCs treated with (A) different concentrations of ox-LDL and (B) $100 \mathrm{mg} / 1 \mathrm{ox}-\mathrm{LDL}$ for 12, $24 \mathrm{or} 48 \mathrm{~h}$. The data are expressed as the mean \pm SEM from three independent experiments. ${ }^{*} \mathrm{P}<0.05,{ }^{* * *} \mathrm{P}<0.01$ and ${ }^{* * * *} \mathrm{P}<0.001$ vs. Control or 0 h. ZFAS1, zinc finger antisense 1 ; ox-LDL, oxidized low-density lipoprotein; VSMCs, vascular smooth muscle cells.

after transfection with or without oe-ZFAS1 or si-ZFAS1, the VSMCs were resuspended in $200 \mu 1$ serum-free DMEM, and $4 \times 10^{4}$ cells were loaded into the upper chambers of Transwell plates precoated with Matrigel Mix for $5 \mathrm{~h}$ at $37^{\circ} \mathrm{C}$ (BD Biosciences), and the lower chambers were filled with $500 \mu 1$ DMEM with $10 \%$ FBS as a chemoattractant. After incubation for $24 \mathrm{~h}$ at $37^{\circ} \mathrm{C}$, the membrane was fixed with $4 \%$ paraformaldehyde for $25 \mathrm{~min}$ at room temperature, and the cells on the lower surface of the membrane were stained with $0.1 \%$ crystal violet solution for $30 \mathrm{~min}$ at room temperature, and finally examined under a fluorescence microscope at x100 magnification (Olympus IX53; Olympus Corporation). The invasion rate was calculated according to the following formula: Number of cells in tested group / number of cells in control group.

Statistical analysis. Statistical analyses were performed using SPSS 19.0 (IBM Corp.), and data are presented as the mean \pm SEM from at least three repeated experiments. Differences between multiple groups were analyzed by one-way ANOVA followed by Tukey's post hoc test. $\mathrm{P}<0.05$ was considered to indicate a statistically significant difference.

\section{Results}

ZFAS1 expression is upregulated by ox-LDL treatment in VSMCs. To verify the role of ZFAS1 in atherosclerosis, VSMCs were incubated with ox-LDL to simulate the high blood lipid environment, and ZFAS1 mRNA expression was detected by RT-qPCR. The results revealed that ZFAS1 mRNA expression was significantly higher with increasing doses of ox-LDL compared with the control group, and the increase was dose-dependent (Fig. 1A). Hence, $100 \mathrm{mg} / \mathrm{l} \mathrm{ox}-\mathrm{LDL}$ was selected for further experimentation. Moreover, VSMCs were stimulated with ox-LDL $(100 \mathrm{mg} / \mathrm{l})$ for 12, 24 and $48 \mathrm{~h}$. The results of RT-qPCR revealed that ZFAS1 mRNA expression was significantly increased by
ox-LDL stimulation at 24 and $48 \mathrm{~h}$ in a time-dependent manner (Fig. 1B). Thus, VSMCs treated with $100 \mathrm{mg} / \mathrm{l}$ ox-LDL for $48 \mathrm{~h}$ were selected for subsequent experiments.

ZFAS1-knockdown inhibits the ox-LDL-induced excessive proliferation of VSMCs. To explore the effect of ZFAS1 expression on cellular behaviors, ZFAS1 expression was knocked down, and the proliferation of VSMCs was analyzed. First, si-ZFAS1 was used to achieve ZFAS1-knockdown. The RT-qPCR results demonstrated that ZFAS1 mRNA expression in the si-ZFAS1-1 and si-ZFAS1-2 groups was significantly lower compared with that in the control group, particularly in the si-ZFAS1-2 group (Fig. 2A). Hence, si-ZFAS1-2 was selected for further experiments. The CCK- 8 assay results indicated that ox-LDL treatment significantly increased the viability of VSMCs at 24 and $48 \mathrm{~h}$ compared with the control group, while ZFAS1-knockdown significantly decreased VSMC viability when compared with the ox-LDL group (Fig. 2B). Finally, the expression levels of proteins associated with cell proliferation was quantified by western blotting. PCNA and Ki67 were identified as proliferation markers. As shown in Fig. 2C, ox-LDL stimulation caused significant upregulation of Ki67 and PCNA expression. Notably, ZFAS1-knockdown in ox-LDL-treated cells led to a significant decrease in Ki67 and PCNA expression (Fig. 2C). Hence, these results indicated that ZFAS1-knockdown inhibited the ox-LDL-induced excessive proliferation of VSMCs.

ZFAS1 overexpression promotes the proliferation of VSMCs stimulated by ox-LDL. To explore the effect of ZFAS1 expression on cell proliferation, VSMC proliferation was determined following ZFAS1 overexpression. The RT-qPCR results revealed that ZFAS1 mRNA expression in VSMCs was significantly increased by oe-ZFAS1 transfection (Fig. 3A). The CCK-8 assay results revealed that ZFAS1 overexpression significantly increased the viability of VSMCs induced by ox-LDL (Fig. 3B). Finally, the western blotting results demonstrated that ZFAS1 overexpression significantly upregulated Ki67 and PCNA expression in ox-LDL-induced VSMCs 
A

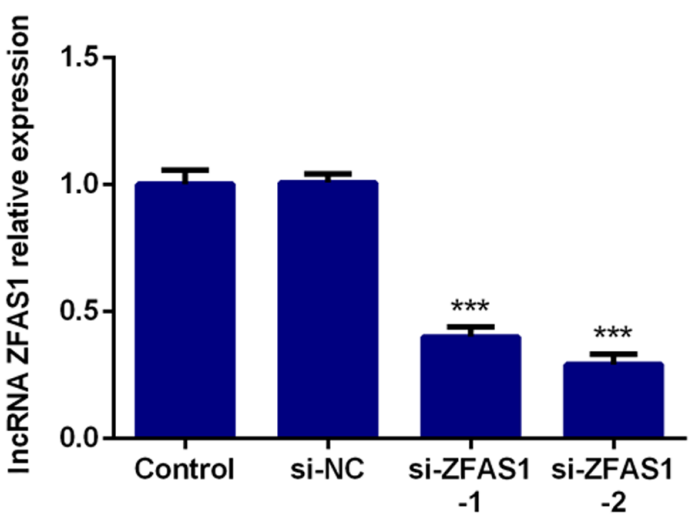

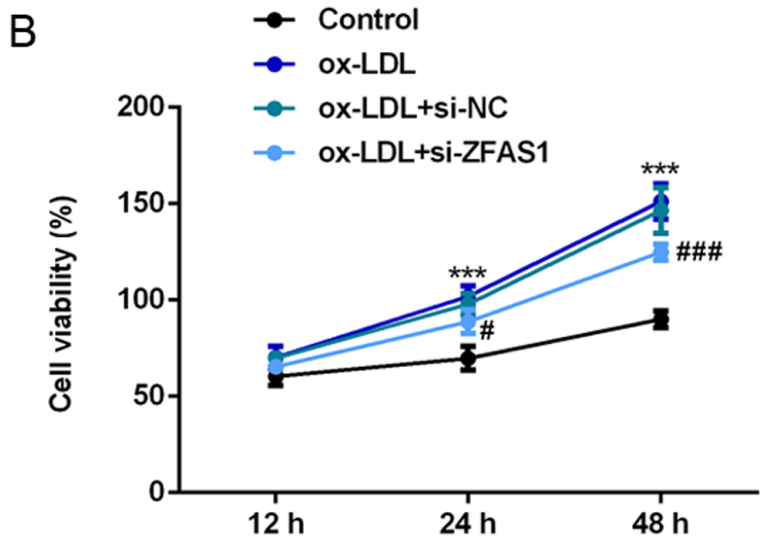
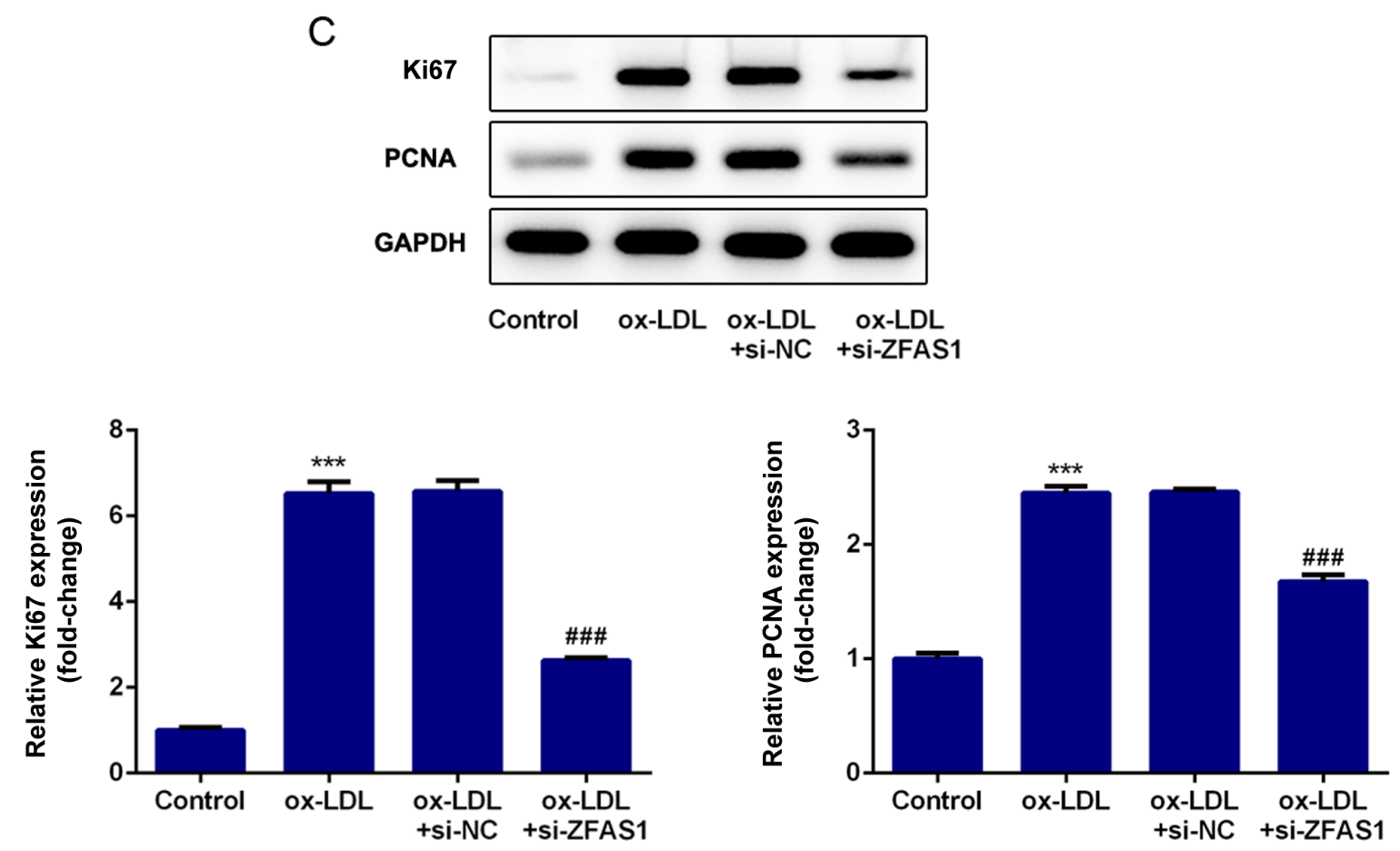

Figure 2. ZFAS1-knockdown inhibits the ox-LDL-induced excessive proliferation of VSMCs. (A) Relative mRNA ZFAS1 expression was quantified via reverse transcription-quantitative PCR following ZFAS1-knockdown. (B) Viability of VSMCs was examined using the Cell Counting Kit-8 assay. (C) Protein expression levels of Ki67 and PCNA were determined by western blotting. The data are expressed as the mean \pm SEM from three independent experiments. ${ }^{* * *} \mathrm{P}<0.001$ vs. Control; ${ }^{\#} \mathrm{P}<0.05$ and ${ }^{\# \# \#} \mathrm{P}<0.001$ vs. ox-LDL + si-NC. ZFAS1, zinc finger antisense 1 ; ox-LDL, oxidized low-density lipoprotein; VSMCs, vascular smooth muscle cells; PCNA, proliferating cell nuclear antigen; si, small interfering RNA; NC, negative control.

(Fig. 3C). Thus, ZFAS1 overexpression promoted the proliferation of VSMCs stimulated by ox-LDL.

ZFAS1-knockdown suppresses the excessive migration and invasion of VSMCs induced by ox-LDL. To investigate the effect of ZFAS1 expression on cellular behavior, ZFAS1 expression was inhibited, and the migration and invasion of VSMCs were analyzed. The results of the wound healing assay revealed that ox-LDL treatment significantly promoted the migration of VSMCs, while ZFAS1-knockdown significantly suppressed the migration of ox-LDL-induced VSMCs (Fig. 4A and C). Furthermore, the results of the Transwell chamber assay demonstrated that ox-LDL stimulation significantly promoted the invasion of VSMCs, whereas ZFAS1-knockdown significantly inhibited the invasion of ox-LDL-treated VSMCs (Fig. 4B and D). Additionally, the expression levels of proteins associated with migration and invasion were quantified. MMP2 and MMP9 are involved in cell migration and invasion. The western blotting results revealed that the expression levels of MMP2 and MMP9 in the ox-LDL and ox-LDL + si-NC groups were significantly higher compared with that in the control group (Fig. 5). Notably, ZFAS1-knockdown significantly inhibited the expression levels of MMP2 and MMP9 following ox-LDL stimulation (Fig. 5). These results indicated that ZFAS1-knockdown suppressed the excessive migration and invasion of VSMCs induced by ox-LDL.

ZFAS1 overexpression promotes the migration and invasion of VSMCs induced by ox-LDL. To investigate the effect of ZFAS1 expression on cell migration and invasion, VSMC migration and invasion were determined following ZFAS1 

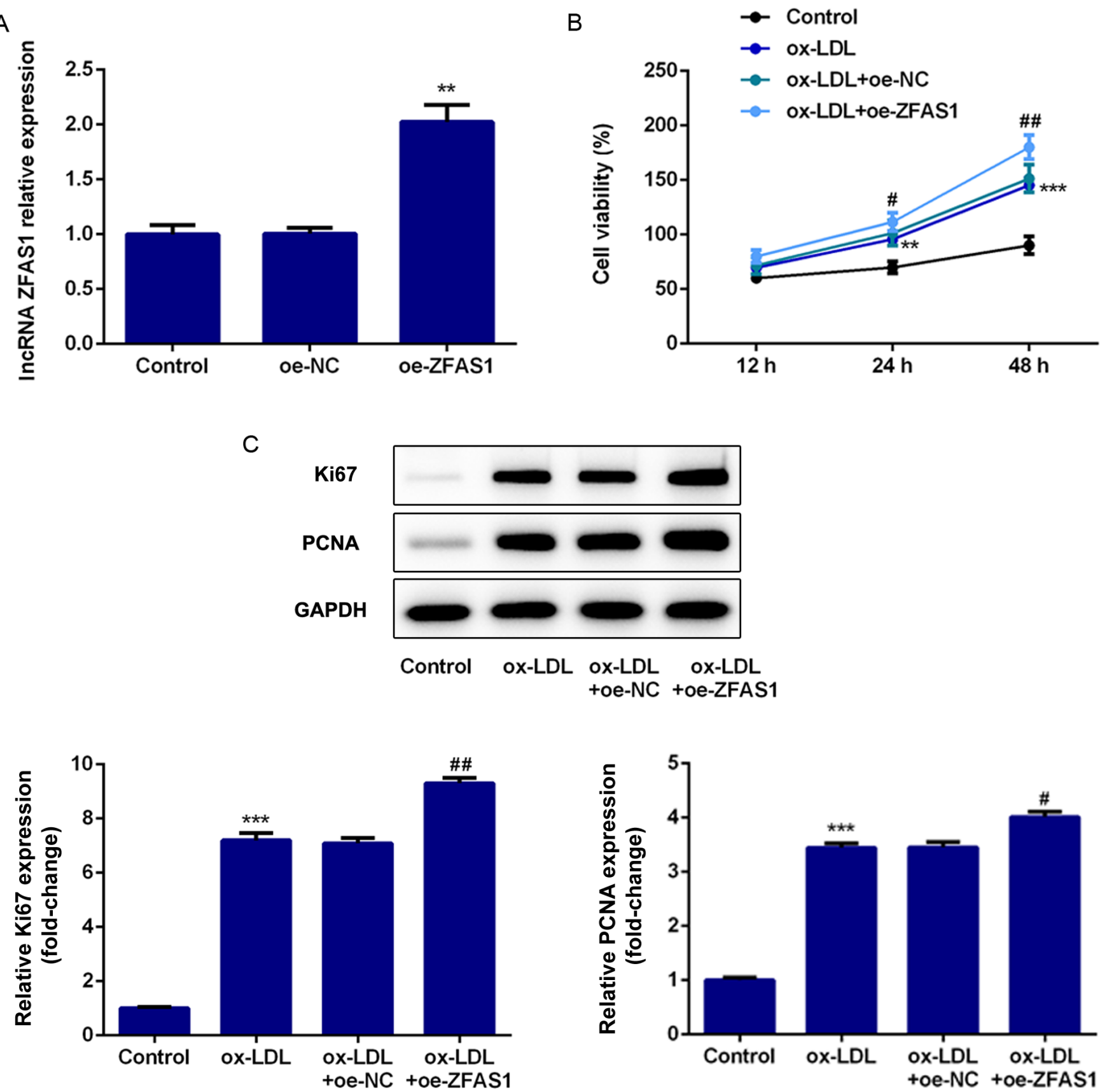

Figure 3.ZFAS1 overexpression promotes the proliferation of VSMCs stimulated by ox-LDL. (A) Relative mRNA ZFAS1 expression was quantified via reverse transcription-quantitative PCR following ZGAS1 overexpression. (B) Viability of VSMCs was examined using the Cell Counting Kit-8 assay. (C) Protein expression levels of Ki67 and PCNA were determined by western blotting. The data are expressed as the mean \pm SEM from three independent experiments. ${ }^{* *} \mathrm{P}<0.01$ and ${ }^{* * *} \mathrm{P}<0.001$ vs. Control; ${ }^{*} \mathrm{P}<0.05$ and ${ }^{\# \#} \mathrm{P}<0.01$ vs. ox-LDL + oe-NC. ZFAS1, zinc finger antisense 1; ox-LDL, oxidized low-density lipoprotein; VSMCs, vascular smooth muscle cells; PCNA, proliferating cell nuclear antigen; oe, overexpression; NC, negative control.

overexpression. As shown in Fig. 6, ZFAS1 overexpression significantly promoted the migration and invasion of ox-LDL-induced VSMCs. Moreover, ZFAS1 overexpression significantly increased MMP2 and MMP9 expression (Fig. 7). These results suggested that ZFAS1 overexpression promoted the migration and invasion of VSMCs induced by ox-LDL.

\section{Discussion}

Atherosclerosis is characterized by endothelium dysfunction, accumulation of ox-LDL and intimal hyperplasia (20).
A previous study has reported that the excessive proliferation and migration of VSMCs in response to vascular injury, inflammation and lipoprotein accumulation mainly leads to the initiation of intimal hyperplasia (21). Hence, VSMCs were treated with ox-LDL to simulate the high blood lipid environment in the present study, which focused on the functional role of ZFAS1 in the behavior of ox-LDL-induced VSMCs. The current findings demonstrated that ox-LDL treatment significantly increased ZFAS1 mRNA expression in VSMCs in a dose- and time-dependent manner, suggesting that ZFAS1 expression is closely associated with the pathogenesis 
A

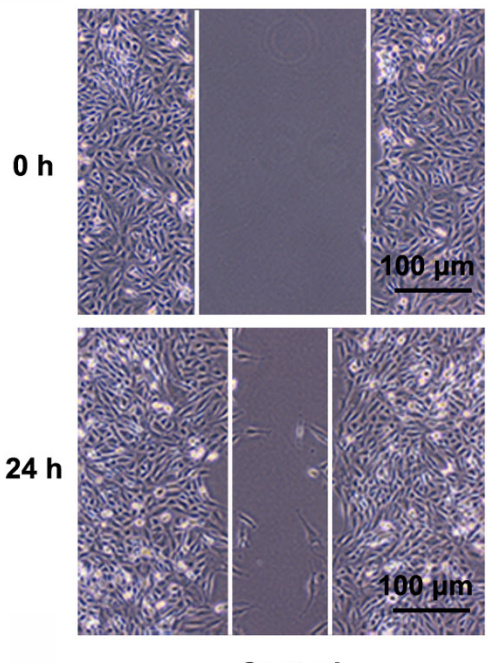

B
ox-LDL
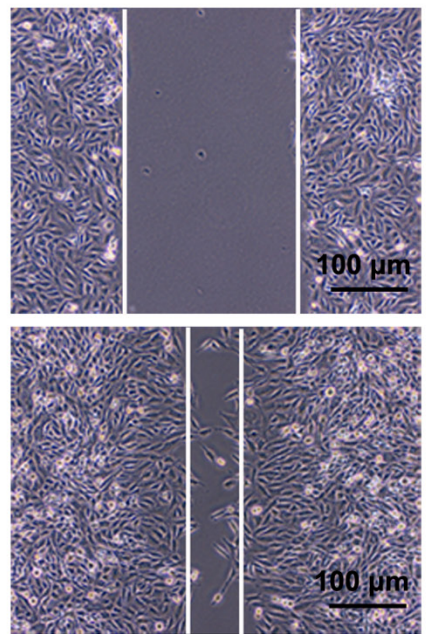

ox-LDL
ox-LDL+si-NC

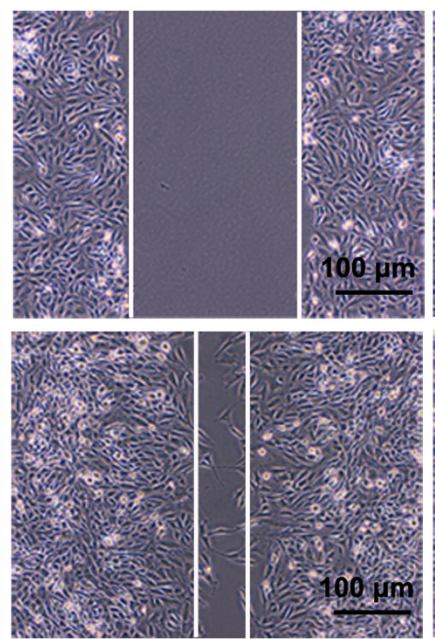

ox-LDL+si-NC
ox-LDL+si-ZFAS1

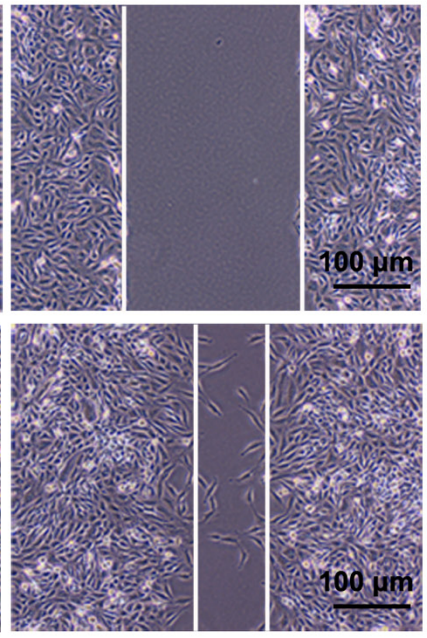

ox-LDL+si-ZFAS1

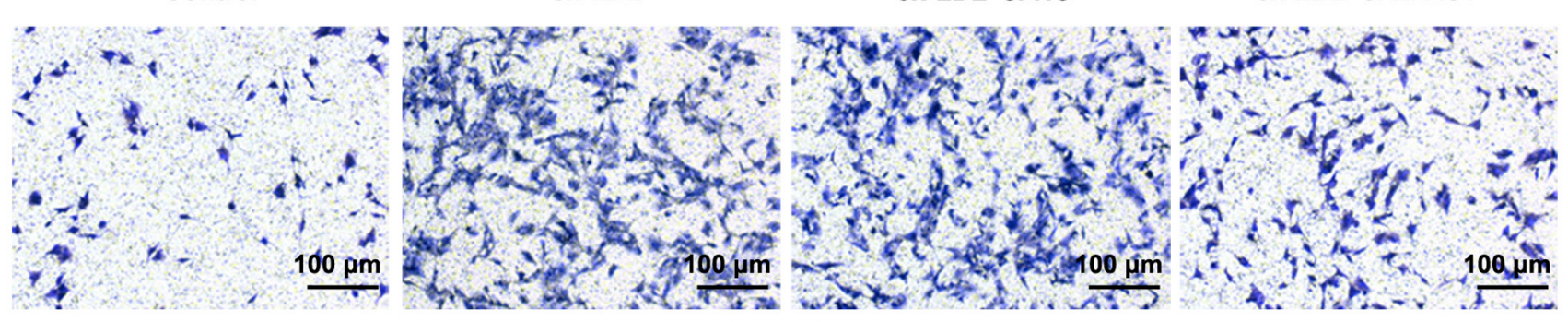

C

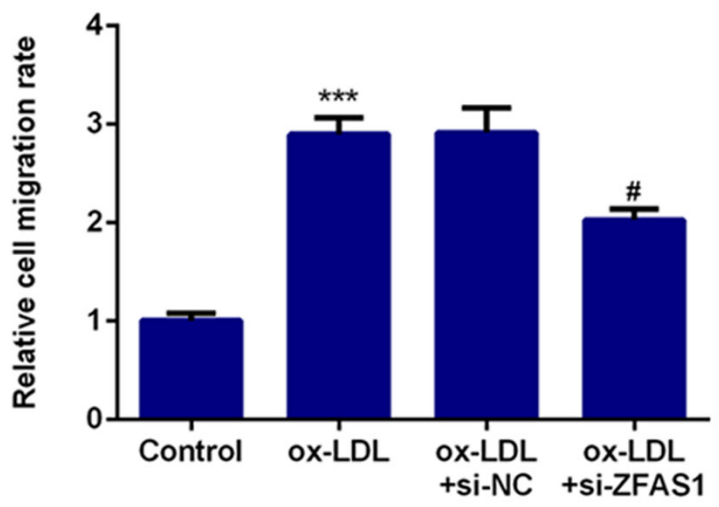

D

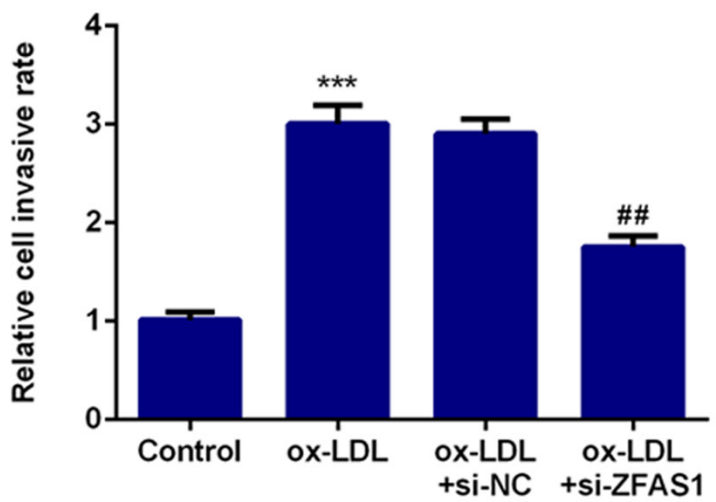

Figure 4. ZFAS1-knockdown suppresses the excessive migration and invasion of VSMCs induced by ox-LDL. (A) Migration of VSMCs was determined by wound healing assay (scale bar, $100 \mu \mathrm{m}$ ). (B) Invasion of VSMCs was determined by Transwell chamber assay (scale bar, $100 \mu \mathrm{m}$ ). (C) Quantification of cell migration. (D) Quantification of cell invasion. The data are expressed as the mean \pm SEM from three independent experiments. ${ }^{* * *} \mathrm{P}<0.001 \mathrm{vs}$. Control; ${ }^{\#} \mathrm{P}<0.05$ and ${ }^{\# \#} \mathrm{P}<0.01$ vs. ox-LDL + si-NC. ZFAS1, zinc finger antisense 1; ox-LDL, oxidized low-density lipoprotein; VSMCs, vascular smooth muscle cells; si, small interfering RNA; NC, negative control.

of atherosclerosis. The aim of the present study was to verify the effect of ZFAS1 on the behavior of ox-LDL-induced VSMCs.

Multiple lncRNAs have recently emerged as regulators of different processes in cardiovascular diseases (22-24). ZFAS1 has been reported as a key lncRNA in atherosclerosis (12). Moreover, ZFAS1 attenuates the rate of cholesterol efflux and facilitates inflammatory responses in atherosclerosis (25). Additionally, ZFAS1 has been found to be dysregulated in various types of cancer and to serve an oncogenic role in the onset and development of malignant tumors, such as ovarian cancer and glioma, by promoting cancer metastasis, growth and epithelial-to-mesenchymal transition (26-28). However, the functional effect of ZFAS1 on cell migration and the proliferation of VSMCs has yet to be fully elucidated. PCNA and Ki67 are identified as cell proliferation markers $(29,30)$. Moreover, it has been reported that MMP2 and MMP9 are associated with cancer metastasis, angiogenesis and invasion $(31,32)$. In the present study, it was observed that ZFAS1 overexpression promoted the ox-LDL-induced proliferation, migration and invasion of VSMCs, and upregulated the expression levels of proteins associated with cell proliferation, migration and invasion (Ki67, PCNA, MMP2 and MMP9). Notably, ZFAS1-knockdown partly reversed the effect of ox-LDL treatment on the proliferation, migration and invasion of VSMCs, and the expression levels of proteins 

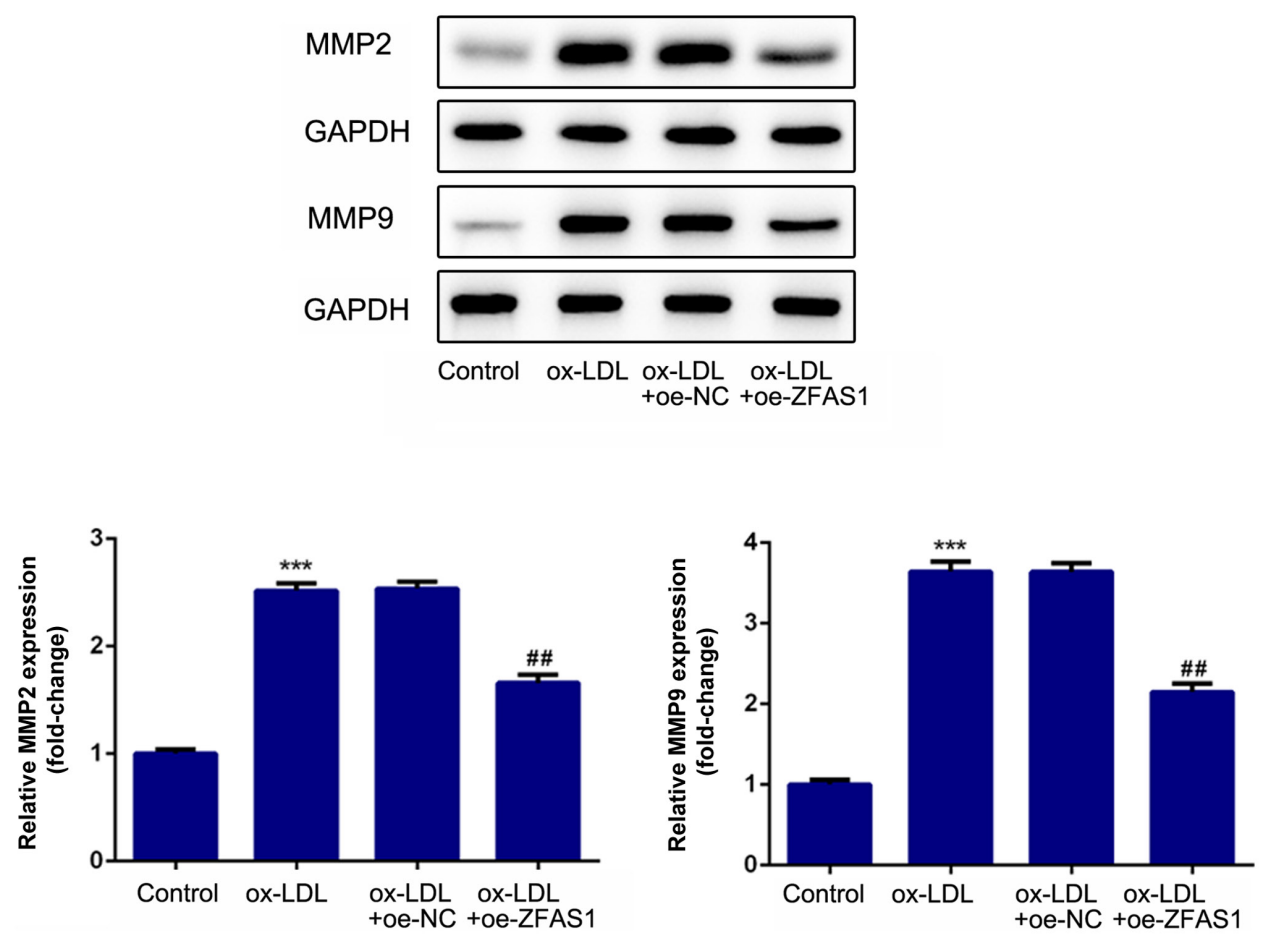

Figure 5. ZFAS1-knockdown suppresses the expression levels of proteins associated with migration and invasion in vascular smooth muscle cells induced by ox-LDL. Protein expression levels of MMP2 and MMP9 were detected by western blotting. The data are expressed as the mean \pm SEM from three independent experiments. ${ }^{* * *} \mathrm{P}<0.001$ vs. Control; ${ }^{\# \#} \mathrm{P}<0.01$ vs. ox-LDL + si-NC. ZFAS1, zinc finger antisense 1; ox-LDL, oxidized low-density lipoprotein; MMP, matrix metallopeptidase; si, small interfering RNA; NC, negative control.

A

Control

ox-LDL

ox-LDL+oe-NC

ox-LDL+oe-ZFAS1
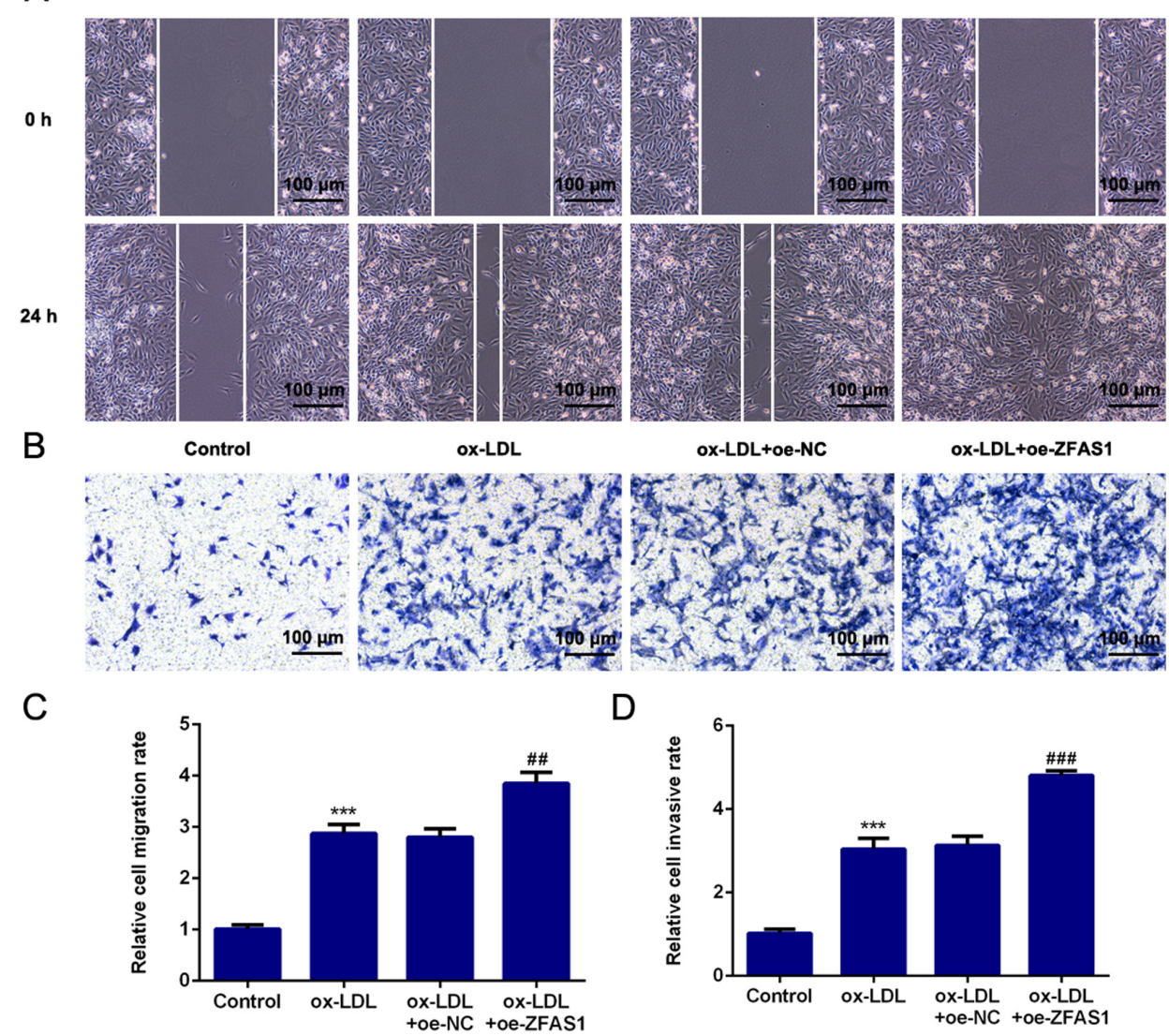

ox-LDL+oe-NC

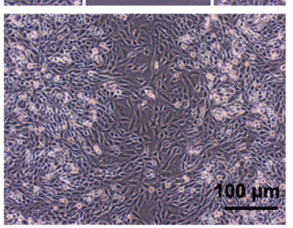

ox-LDL+oe-ZFAS1

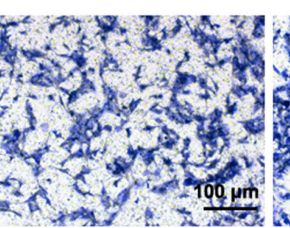

(2)

D

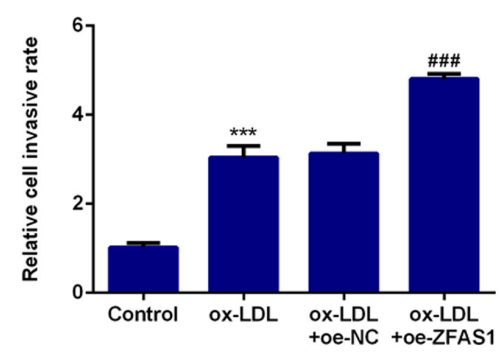

Figure 6. ZFAS1 overexpression promotes the migration and invasion of VSMCs induced by ox-LDL. (A) Migration of VSMCs was determined by the wound healing assay (scale bar, $100 \mu \mathrm{m}$ ). (B) Invasion of VSMCs was determined by the Transwell chamber assay (scale bar, $100 \mu \mathrm{m}$ ). (C) Quantification of cell migration. (D) Quantification of cell invasion. The data are expressed as the mean \pm SEM from three independent experiments. ${ }^{* * *} \mathrm{P}<0.001$ vs. Control; ${ }^{\# \#} \mathrm{P}<0.01$ and ${ }^{\# \#} \mathrm{P}<0.001$ vs. ox-LDL + oe-NC. ZFAS1, zinc finger antisense 1; ox-LDL, oxidized low-density lipoprotein; VSMCs, vascular smooth muscle cells; oe, overexpression; NC, negative control. 

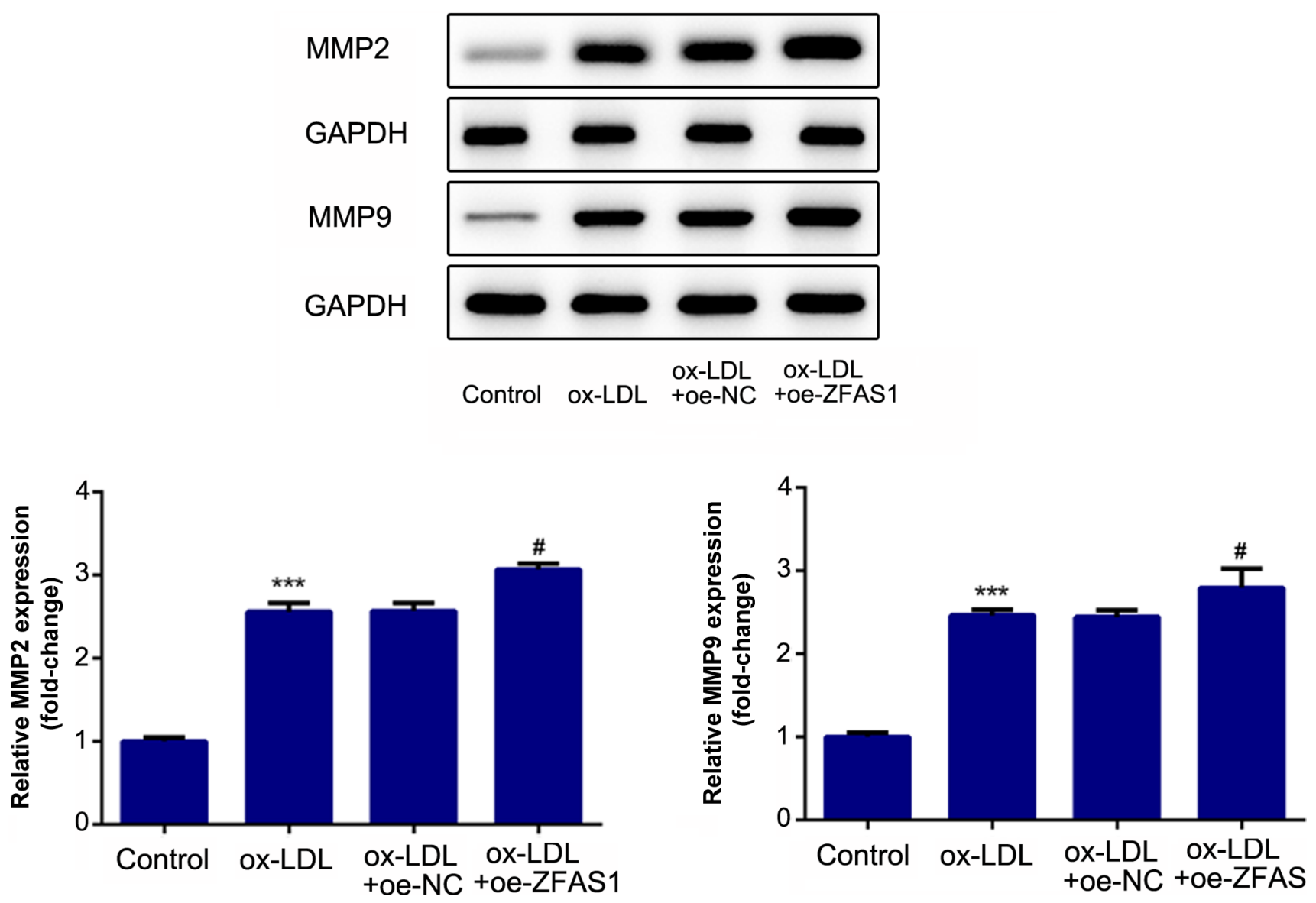

Figure 7. ZFAS1-knockdown increases the expression levels of proteins associated with migration and invasion in vascular smooth muscle cells induced by ox-LDL. Protein expression levels of MMP2 and MMP9 were detected by western blotting. The data are expressed as the mean \pm SEM from three independent experiments. ${ }^{* * *} \mathrm{P}<0.001$ vs. Control; " $\mathrm{P}<0.05$ vs. ox-LDL + oe-NC. ZFAS1, zinc finger antisense 1; ox-LDL, oxidized low-density lipoprotein; MMP, matrix metallopeptidase; oe, overexpression; NC, negative control.

associated with cell proliferation, migration and invasion. Previous studies have demonstrated that the phenotype of VSMCs can be switched from contractile to proliferative and migratory during the process of atherosclerosis $(33,34)$. Furthermore, inhibiting cell proliferation, migration and invasion may serve as an effective therapeutic strategy for preventing cardiovascular disease (35). Thus, the present findings indicated that ZFAS1 may serve an important regulatory role in the phenotypic transition of ox-LDL-induced VSMCs. Overall, the aforementioned findings indicated that ZFAS1 promoted the ox-LDL-induced proliferation, invasion and migration of VSMCs, and may serve as a potential biomarker for the dysfunction of VSMCs in the pathological condition of atherosclerosis. However, there are some limitations in the present study, since the conclusion was only from the results of in vitro experiments. It is necessary to further investigate the role of ZFAS1 in vivo to confirm the findings of the present study. Hence, further studies should be performed to confirm whether ZFAS1 may be used as a biomarker and therapeutic target of atherosclerosis.

In conclusion, the findings of the present study suggested that ZFAS1 expression was upregulated by ox-LDL stimulation in VSMCs. Moreover, ZFAS1 promoted the ox-LDL-induced proliferation, invasion and migration of VSMCs, as well as the expression levels of Ki67, PCNA,
MMP2 and MMP9, and may represent a novel biomarker for dysfunction of VSMCs in the pathological condition of atherosclerosis.

\section{Acknowledgements}

Not applicable.

\section{Funding}

No funding was received.

\section{Availability of data and materials}

The datasets used and/or analyzed during the current study are available from the corresponding author on reasonable request.

\section{Authors' contributions}

HW, HH, JM, YJ and RC conceived and designed the study, collected, analyzed and interpreted the data, and revised the manuscript. HW wrote the manuscript. HW and RC confirmed the authenticity of the raw data. All authors read and approved the final manuscript. 


\section{Ethics approval and consent to participate}

Not applicable.

\section{Patient consent for publication}

Not applicable.

\section{Competing interests}

The authors declare that they have no competing interests.

\section{References}

1. Liu M, Song Y and Han Z: Study on the effect of lncRNA AK094457 on OX-LDL induced vascular smooth muscle cells. Am J Transl Res 11: 5623-5633, 2019.

2. Bennett MR, Sinha S and Owens GK: Vascular smooth muscle cells in atherosclerosis. Circ Res 118: 692-702, 2016.

3. Wang J, Uryga AK, Reinhold J, Figg N, Baker L, Finigan A, Gray K, Kumar S, Clarke M and Bennett M: Vascular smooth muscle cell senescence promotes atherosclerosis and features of plaque vulnerability. Circulation 132: 1909-1919, 2015.

4. Misra A, Feng Z, Chandran RR, Kabir I, Rotllan N, Aryal B, Sheikh AQ, Ding L, Qin L, Fernández-Hernando C, et al: Integrin beta3 regulates clonality and fate of smooth muscle-derived atherosclerotic plaque cells. Nat Commun 9: 2073, 2018.

5. Hu D, Yin C, Luo S, Habenicht AJR and Mohanta SK: Vascular smooth muscle cells contribute to atherosclerosis immunity. Front Immunol 10: 1101, 2019.

6. Xu L, Hao H, Hao Y, Wei G, Li G, Ma P, Ding N, Ma S, Chen AF and Jiang Y: Aberrant MFN2 transcription facilitates homocysteine-induced VSMCs proliferation via the increased binding of c-Myc to DNMT1 in atherosclerosis. J Cell Mol Med 23: 4611-4626, 2019

7. Harman JL and Jørgensen HF: The role of smooth muscle cells in plaque stability: Therapeutic targeting potential. Br J Pharmacol 176: 3741-3753, 2019.

8. Kattoor AJ, Kanuri SH and Mehta JL: Role of Ox-LDL and LOX-1 in atherogenesis. Curr Med Chem 26: 1693-1700, 2019.

9. Huang M, Zhong Z, Lv M, Shu J, Tian Q and Chen J: Comprehensive analysis of differentially expressed profiles of lncRNAs and circRNAs with associated co-expression and ceRNA networks in bladder carcinoma. Oncotarget 7 : 47186-47200, 2016

10. Huang X, Zhi X, Gao Y, Ta N, Jiang $h$ and Zheng J: lncRNAs in pancreatic cancer. Oncotarget 7: 57379-57390, 2016.

11. Mathy NW and Chen XM: Long non-coding RNAs (lncRNAs) and their transcriptional control of inflammatory responses. J Biol Chem 292: 12375-12382, 2017.

12. Wang CH, Shi HH, Chen LH, Li XL, Cao GL and Hu XF: Identification of key lncRNAs associated with atherosclerosis progression based on public datasets. Front Genet 10: 123 2019.

13. Chen L, Yao H, Hui JY, Ding SH, Fan YL, Pan YH, Chen KH, Wan JQ and Jiang JY: Global transcriptomic study of atherosclerosis development in rats. Gene 592: 43-48, 2016.

14. Zhang Y, Jiao L, Sun L, Li Y, Gao Y, Xu C, Shao Y, Li M, Li C, Lu Y, et al: lncRNA ZFAS1 as a SERCA2a inhibitor to cause intracellular $\mathrm{Ca}^{2+}$ overload and contractile dysfunction in a mouse model of myocardial infarction. Circ Res 122: 1354-1368, 2018.

15. Wang X, Jin Q, Chen W and Cai Z: lncRNA ZFAS1 promotes proliferation and migration and inhibits apoptosis in nasopharyngeal carcinoma via the PI3K/AKT pathway in vitro. Cancer Biomark 26: 171-182, 2019

16. Meng Q, Zhang R, Ding W and Mao B: Long noncoding RNA ZFAS1 promotes cell proliferation and tumor growth by upregulating LIN28 in cervical carcinoma. Minerva Med 111: 511-514, 2019.

17. Xie S, Ge Q, Wang X, Sun X and Kang Y: Long non-coding RNA ZFAS1 sponges miR-484 to promote cell proliferation and invasion in colorectal cancer. Cell Cycle 17: 154-161, 2018.
18. Ye D, Jian W, Feng J and Liao X: Role of long noncoding RNA ZFAS1 in proliferation, apoptosis and migration of chondrocytes in osteoarthritis. Biomed Pharmacother 104: 825-831, 2018.

19. Livak KJ and Schmittgen TD: Analysis of relative gene expression data using real-time quantitative PCR and the 2(-Delta Delta C(T)) method. Methods 25: 402-408, 2001.

20. Spartalis M, Spartalis E, Athanasiou A, Paschou SA, Kontogiannis C, Georgiopoulos G, Iliopoulos DC and Voudris V: The role of the endothelium in premature atherosclerosis: Molecular mechanisms. Curr Med Chem 27: 1041-1051, 2020.

21. Zhao XS, Zheng B, Wen Y, Sun Y, Wen JK and Zhang XH: Salvianolic acid B inhibits Ang II-induced VSMC proliferation in vitro and intimal hyperplasia in vivo by downregulating miR-146a expression. Phytomedicine 58: 152754, 2019.

22. Guo FX, Wu Q, Li P, Zheng L, Ye S, Dai XY, Kang CM, Lu JB, $\mathrm{Xu} \mathrm{BM}, \mathrm{Xu}$ YJ, et al: The role of the lncRNA-FA2H-2-MLKL pathway in atherosclerosis by regulation of autophagy flux and inflammation through mTOR-dependent signaling. Cell Death Differ 26: 1670-1687, 2019

23. Li FP, Lin DQ and Gao LY: lncRNA TUG1 promotes proliferation of vascular smooth muscle cell and atherosclerosis through regulating miRNA-21/PTEN axis. Eur Rev Med Pharmacol Sci 22: 7439-7447, 2018.

24. Ye ZM, Yang S, Xia YP, Hu RT, Chen S, Li BW, Chen SL, Luo XY, Mao L, Li Y, et al: lncRNA MIAT sponges miR-149-5p to inhibit efferocytosis in advanced atherosclerosis through CD47 upregulation. Cell Death Dis 10: 138, 2019.

25. Tang $X$, Yin $R$, Shi $H$, Wang $X$, Shen $D$ and Pan $C$ : lncRNA ZFAS1 confers inflammatory responses and reduces cholesterol efflux in atherosclerosis through regulating miR-654-3p-ADAM10/RAB22A axis. Int J Cardiol 315: 72-80, 2020.

26. Han S, Li DZ and Xiao MF: lncRNA ZFAS1 serves as a prognostic biomarker to predict the survival of patients with ovarian cancer. Exp Ther Med 18: 4673-4681, 2019.

27. Li X, Luo Y, Liu L, Cui S, Chen W, Zeng A, Shi Y and Luo L: The long noncoding RNA ZFAS1 promotes the progression of glioma by regulating the miR-150-5p/PLP2 axis. J Cell Physiol 235: 2937-2946, 2020

28. Zeng Z, Zhao G, Rao C, Hua G, Yang M, Miao X, Ying J and Nie L: Knockdown of lncRNA ZFAS1-suppressed non-small cell lung cancer progression via targeting the miR-150-5p/HMGA2 signaling. J Cell Biochem: Nov 6, 2019 (Epub ahead of print). doi: 10.1002/jcb.29542.

29. Ben-Izhak O, Bar-Chana M, Sussman L, Dobiner V, Sandbank J, Cagnano M, Cohen h and Sabo E: Ki67 antigen and PCNA proliferation markers predict survival in anorectal malignant melanoma. Histopathology 41: 519-525, 2002.

30. Li N, Deng W, Ma J, Wei B, Guo K, Shen W, Zhang Y and Luo S: Prognostic evaluation of Nanog, Oct4, Sox2, PCNA, Ki67 and E-cadherin expression in gastric cancer. Med Oncol 32: 433, 2015.

31. Farina P, Tabouret E, Lehmann P, Barrie M, Petrirena G, Campello C, Boucard C, Graillon T, Girard N and Chinot O: Relationship between magnetic resonance imaging characteristics and plasmatic levels of MMP2 and MMP9 in patients with recurrent high-grade gliomas treated by Bevacizumab and Irinotecan. J Neurooncol 132: 433-437, 2017.

32. Struckmann K, Mertz K, Steu S, Storz M, Staller P, Krek W, Schraml P and Moch H: pVHL co-ordinately regulates CXCR4/CXCL12 and MMP2/MMP9 expression in human clear-cell renal cell carcinoma. J Pathol 214: 464-471, 2008.

33. Gomez D and Owens GK: Smooth muscle cell phenotypic switching in atherosclerosis. Cardiovasc Res 95: 156-164, 2012.

34. Wei M, Liu Y, Zheng M, Wang L, Ma F, Qi Y and Liu G: Upregulation of protease-activated receptor 2 promotes proliferation and migration of human vascular smooth muscle cells (VSMCs). Med Sci Monit 25: 8854-8862, 2019.

35. Luo Z, Deng H, Fang Z, Zeng A, Chen Y, Zhang W and Lu Q: Ligustilide inhibited rat vascular smooth muscle cells migration via c-Myc/MMP2 and ROCK/JNK signaling pathway. J Food Sci 84: 3573-3583, 2019

This work is licensed under a Creative Commons Attribution-NonCommercial-NoDerivatives 4.0 International (CC BY-NC-ND 4.0) License. 